\title{
Effects of rehabilitation programs on heart rate variability after stroke: a systematic review
}

\author{
Efeitos dos programas de reabilitação na variabilidade da frequência cardíaca após \\ acidente vascular cerebral: uma revisão sistemática
}

Thais Regina BELLI', Luciane Aparecida Pascucci Sande de SOUZA', Silméia Garcia Zanati BAZAN², Rodrigo BAZAN, Gustavo José LUVIZUTTO'1

\begin{abstract}
Background: It has been shown that the autonomic nervous system can be modulated by physical exercise after stroke, but there is a lack of evidence showing rehabilitation can be effective in increasing heart rate variability (HRV). Objective: To investigate the effectiveness and safety of rehabilitation programs in modulating HRV after stroke. Methods: The search strategy was based in the PICOT (patients: stroke; interventions: rehabilitation; comparisons: any control group; outcomes: HRV; time: acute, subacute and chronic phases of stroke). We searched MEDLINE, CENTRAL, CINAHL, LILACS, and SCIELO databases without language restrictions, and included randomized controlled trials (RCTs), quasi-randomized controlled trials (quasi-RCTs), and non-randomized controlled trials (non-RCTs). Two authors independently assessed the risk of bias and we used the Grading of Recommendations Assessment, Development and Evaluation (GRADE) methodology to rate the certainty of the evidence for each included study. Results: Four studies (two RCTs with low certainty of the evidence and two non-RCTs with very low certainty of the evidence) were included. Three of them showed significant cardiac autonomic modulation during and after stroke rehabilitation: LF/HF ratio (low frequency/high frequency) is higher during early mobilization; better cardiac autonomic balance was observed after body-mind interaction in stroke patients; and resting SDNN (standard deviation of normal R-R intervals) was significantly lower among stroke patients indicating less adaptive cardiac autonomic control during different activities. Conclusions: There are no definitive conclusions about the main cardiac autonomic repercussions observed in post-stroke patients undergoing rehabilitation, although all interventions are safe for patients after stroke.
\end{abstract}

Keywords: Stroke; Heart Rate; Rehabilitation; Physical Therapy.

\section{RESUMO}

Antecedentes: O sistema nervoso autônomo pode ser modulado pelo exercício físico após o acidente vascular cerebral (AVC), mas faltam evidências que demonstrem que a reabilitação pode ser eficaz no aumento da variabilidade da frequência cardíaca (VFC). Objetivo: Investigar a eficácia e segurança dos programas de reabilitação na modulação da VFC após o AVC. Métodos: A estratégia de busca foi baseada na estratégia PICOT (pacientes: AVC; intervenções: reabilitação; comparações: qualquer grupo de controle; desfechos: VFC; tempo: fase aguda, subaguda e crônica). Foi realizada busca nas bases MEDLINE, CENTRAL, CINAHL, LILACS e SCIELO sem restrições de idioma, sendo incluídos ensaios clínicos randomizados (ECRs), ensaios clínicos quasi-randomizados (quasi-ECRs) e ensaios clínicos nãorandomizados (não-ECRs). Dois autores avaliaram independentemente o risco de viés e a metodologia GRADE para classificar a certeza das evidências para cada estudo incluído. Resultados: Quatro estudos (dois ECRs com baixa certeza de evidência e dois não-ECRs com muito baixa certeza de evidência) foram incluídos. Três deles apresentaram modulação autonômica cardíaca durante e após a reabilitação: a razão LF/HF (low frequency/high frequency) foi maior durante a mobilização precoce; maior equilíbrio autonômico foi observado após prática de interação corpo-mente após AVC; e SDNN (desvio padrão dos intervalos R-R normais) foi significativamente menor em pacientes

\footnotetext{
1 Universidade Federal do Triângulo Mineiro, Departamento de Fisioterapia Aplicada, Uberaba MG, Brazil.

Universidade Estadual Paulista, Faculdade de Medicina de Botucatu, Departamento de Clínica Médica, Botucatu SP, Brazil.

${ }^{3}$ Universidade Estadual Paulista, Departamento de Neurologia, Psicologia e Psiquiatria, Botucatu SP, Brazil.

TRB (D) https://orcid.org/0000-0001-7603-298X; LAPSS (D) http://orcid.org/0000-0002-7160-9556; SGZB (D) http://orcid.org/0000-0002-0607-8189; RB (D) https://orcid.org/0000-0003-3872-308X; GJL (D) https://orcid.org/0000-0002-6914-7225

Correspondence: Gustavo José Luvizutto; Email: gluvizutto@gmail.com.

Conflicts of interest: There is no conflict of interest to declare.

Authors' contribution: GJL, TRB: conceiving of the review; GJL, LAPSS : co-ordinating the review; TRB, GJL: undertaking manual searches; TRB, SGZB, RB: screening search results; SGZB, RB: organizing retrieval of papers; GJL: screening retrieved papers against inclusion criteria, analyzing statistical data, making statistical inferences, servind as guarantor for the review (one review author); GJL, RB, LAPSS: appraising quality of papers; TRB, SGZB: extracting data from papers; GJL, RB: Writing to authors of papers for additional information;TRB: providing additional data about papers; TRB, LAPSS, SGZB, RB: obtaining and screening data on unpublished studies, managing data for the review; GJL, RB, SGZB, LAPSS: interpreting data; All authors: writing the review, taking responsibility for reading and checking the review before submission.

Received on September 01, 2020; Received in its final form on November 05, 2020; Accepted on November $17,2020$.
} 
com AVC indicando menor adaptação autonômica cardíaca durante diferentes atividades. Conclusões: Não há conclusão definitivas sobre as principais repercussões cardíaca autonômica nos pacientes com AVC na reabilitação, embora todas as intervenções foram seguras para os pacientes após AVC.

Palavras-chave: Acidente Cascular Cerebral; Variabilidade Cardíaca; Reabilitação; Fisioterapia.

\section{INTRODUCTION}

Stroke is one of the main causes of morbidity and mortality in industrialized countries and the leading cause of chronic disability in adults ${ }^{1-3}$. After stroke, more than $70 \%$ of individuals present alterations in motor, sensory, or cognitive systems, which can be mild and transient or severe and disabling, and these alterations can be related to autonomic nervous system impairments, which can lead to changes in heart rate variability $(\mathrm{HRV})^{4-6}$.

$\mathrm{HRV}$ is the result of adaptive changes in heart rate caused by sympathetic and parasympathetic activity in response to external or internal stimuli? ${ }^{7}$. Based on this concept, HRV is defined as the changes in heart rate (HR) that occur after a stimulus, and it is a predictor of processes related to the autonomic nervous system. Studies have shown that a low HRV response is related to a high risk of stroke ${ }^{8,9}$, severe stroke severity $^{10}$, mortality after stroke ${ }^{4,5,11}$, low vagal modulation ${ }^{12}$, and a poor prognosis after stroke ${ }^{13}$.

There is evidence that physical inactivity reduces cardiac autonomic modulation after stroke ${ }^{14}$. Therefore, the autonomic nervous system can be increased through physical exercise and rehabilitation programs after stroke ${ }^{15}$. Lower HRV is a predictor of morbidity and mortality and cardiac changes increase the risk of death after stroke ${ }^{16}$ and may be related to unfavorable outcomes ${ }^{17}$. Additional studies need to be conducted to elucidate the cardiac autonomic modulating mechanisms and clinical repercussions of HRV after stroke rehabilitation.

Thus, it is possible that specific and effective rehabilitation programs, allowing greater cardiovascular stability, functional gains, and quality of life in individuals after stroke, can be developed. Due to the lack of evidence that rehabilitation can be effective in modulating the autonomic nervous system after stroke, there is no consensus on this effect; there are no systematic reviews in the literature on this topic. Therefore, the aim of this review was to evaluate the effectiveness and safety of rehabilitation programs in modulating HRV after stroke.

\section{METHODS}

We adhered to the methods described in the Cochrane Handbook for Intervention Reviews ${ }^{18}$ and to the Preferred
Reporting Items for Systematic Reviews and Meta-Analyses (PRISMA) reporting guidelines ${ }^{19}$. This review was registered in the International Prospective Register of Systematic Reviews (PROSPERO - CRD42020156527).

\section{Eligibility criteria}

The eligibility criteria were as follows:

1. Study designs: RCTs, quasi-RCTs, and non-RCTs

2. Participants: adults over 18 years of age of either sex with any duration of illness, severity of initial impairment, type of stroke diagnosis (ischemic or intracranial hemorrhage) that was made by a clinical examination or radiographically by computed tomography (CT) or magnetic resonance imaging (MRI).

3. Interventions: any rehabilitation protocol for stroke recovery (early mobilization, physical exercises)

4. Comparators: any conventional stroke rehabilitation program

5. Outcomes: Heart rate variability

\section{Data sources and search strategy}

The search strategy was based in the PICOT (patients: stroke; intervention: rehabilitation; comparison: any control group; outcome: heart rate variability; time: acute, subacute, and chronic phases of stroke). We searched MEDLINE (OvidSP), the Cochrane Central Register of Controlled Trials (CENTRAL), CINAHL, the Latin-American and Caribbean Center on Health Sciences Information (LILACS), and SCIELO databases without language restrictions. The date of the most recent search was July 10, 2020. All searches were conducted with the assistance of a trained medical librarian. We also searched the reference lists of relevant articles and conference proceedings, and contacted the authors of the included trials.

The search terms included "Heart rate variability or (MeSH terms)" and stroke or (MeSH terms) and rehabilitation or (MeSH terms).

\section{Other resources searched}

In an effort to identify additional published, unpublished, and ongoing trials, we performed the following steps: 
- $\quad$ screened the reference lists of the identified studies;

- contacted the study authors and experts; and

- used the Science Citation Index Cited Reference Search to track important articles.

\section{Selection of the studies}

Two pairs of reviewers independently screened all titles and abstracts identified in the literature search, obtained fulltext articles of all the potentially eligible studies, and evaluated the articles for eligibility. The reviewers resolved disagreements by discussion or, if necessary, with third party adjudication. We also considered studies reported only as conference abstracts.

We used the START program (State of the Art through Systematic Review), developed by the Software Engineering Research Laboratory of the Federal University of São Carlos for data organization.

\section{Data extraction}

The reviewers underwent calibration exercises and worked in pairs to independently extract data from the included studies according to the recommendations of the Cochrane Handbook for Systematic Reviews of Interventions ${ }^{20}$. Disagreements were resolved by discussion or, if necessary, with third party adjudication. Reviewers collected the following data using a pretested data extraction form: study design, participants, interventions, comparators, assessed outcomes, and relevant statistical data.

\section{Risk of bias assessment}

Two authors of this review independently assessed the risk of bias for each study using the criteria outlined in the Cochrane Handbook for Systematic Reviews of Interventions ${ }^{20}$. Disagreements were resolved by discussion or by consultation with another review author. We assessed the risk of bias according to the following domains.

- Random sequence generation.

- Allocation concealment.

- Blinding of the participants and personnel.

- Blinding of the outcome assessment.
- Incomplete outcome data.

- Selective outcome reporting.

- $\quad$ Other biases.

We graded the risk of bias for each domain as high, low, or unclear and provided information from the study report, together with justification for our judgment, in the "Risk of bias" tables. For incomplete outcome data in individual studies, we stipulated a low risk of bias for a loss to follow-up of less than $10 \%$ and a difference of less than $5 \%$ in missing data between the intervention/exposure and control groups.

\section{Certainty of evidence}

We summarized the evidence and assessed its certainty separately for bodies of evidence from RCT and nonRCT studies. We used the Grading of Recommendations Assessment, Development and Evaluation (GRADE) methodology to rate the certainty of the evidence for each outcome as high, moderate, low, or very low. In the GRADE approach, RCTs begin with high certainty, and non-RCT studies begin with moderate certainty. Detailed GRADE guidelines were used to assess the overall risk of bias, imprecision, inconsistency, indirectness, and publication bias and to summarize the results in an evidence profile (Table 1$)^{21}$.

We planned to assess publication bias through the visual inspection of funnel plots for each outcome for which we identified 10 or more eligible studies; however, we were not able to do so because there were an insufficient number of studies to conduct this assessment.

\section{Data synthesis and statistical analysis}

It was not possible to perform a meta-analysis due to the non-homogeneity of the interventions. The effects of the interventions, risk of bias, and quality of evidence for each study are reported.

\section{RESULTS}

We identified a total of 88 studies through database searches (see Figure 1 for the search results). After screening the titles and then the abstracts, we obtained full-text articles

Table 1. Characteristics of the included studies.

\begin{tabular}{lcccc}
\hline Author/Year & Study design & N/Age & Stroke time (days) & HRV variables \\
\hline Nozoe et al., 2018 & Non-RCT & $N=21 / 71 y^{*}$ & $1-10$ & LF, InHF and LF/HF \\
Chen et al., 201923 & RCT & $N=72 / 65 \pm 13.5 y$ & $1-10$ & SDNN, LF, HF and LF/HF \\
Beer et al., 2018 ${ }^{24}$ & Non-RCT & $N=19 / 63 y^{*}$ & Post-acute & SDNN and RMSSD \\
Katz-Leurer \& Shochina, 200722 & RCT & $N=64 / 62 \pm 8.5 y$ & 15 & LF and HF \\
\hline
\end{tabular}

RCT: randomized clinical trial; HRV: heart rate variability; LF: low frequency; InHF: natural logarithm of HF power; LF/HF: low to high frequency ratio; SDNN: standard deviation of normal R-R intervals; RMSSD: root-mean-square difference of successive normal R-R intervals; ${ }^{*}$ The authors did not report the standard deviation. 


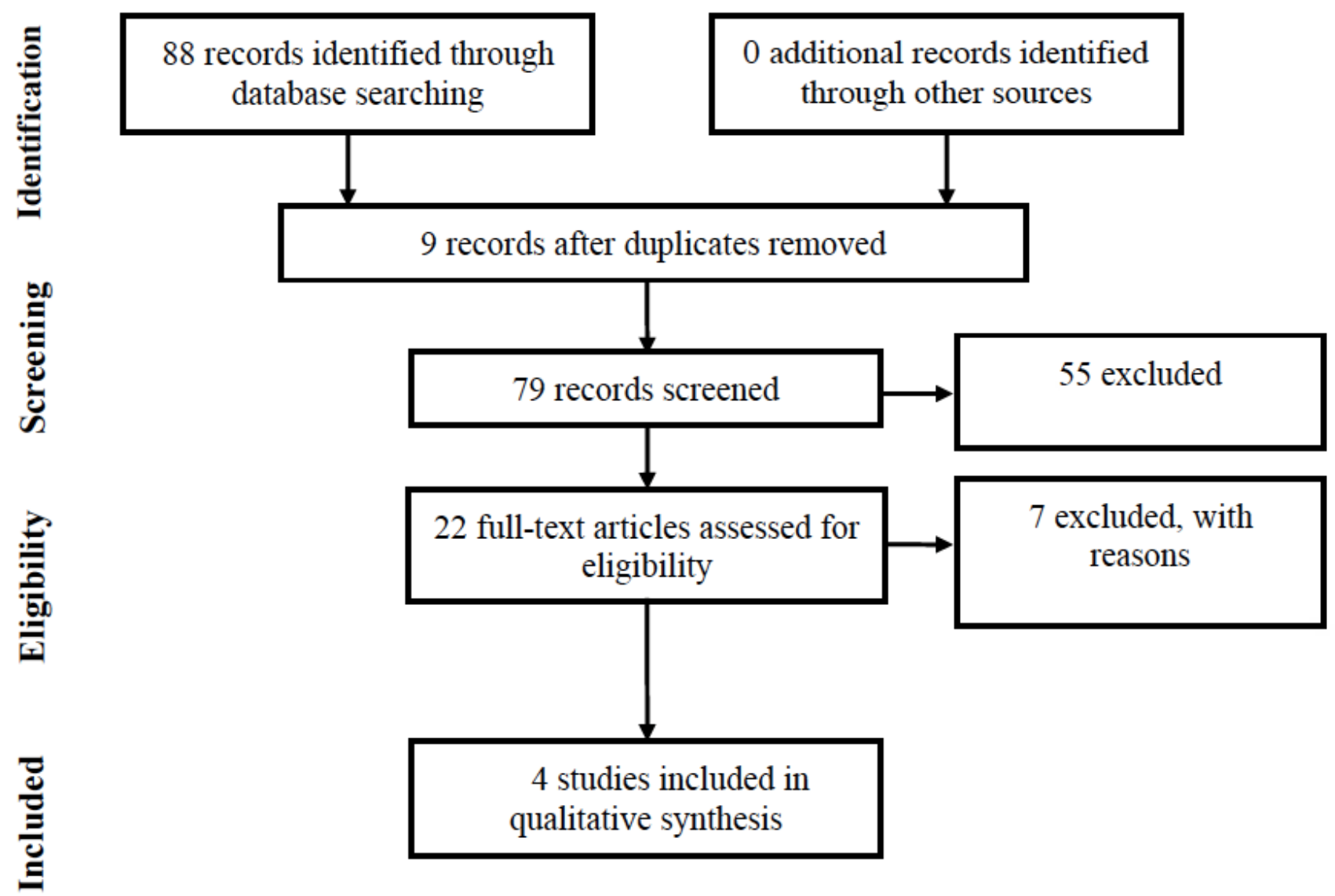

Figure 1. Flow chart of the search results.

for the 22 studies that were potentially eligible for inclusion in the review. We excluded 18 studies because they were considered one of the following types of articles: case report, case series, self-controlled study, review, or a study that was not relevant. The remaining two $\mathrm{RCTs}^{22,23}$ and two non-RCTs ${ }^{15,24}$ were included in this review.

\section{Characteristics of the participants and groups}

All participants in the included studies were diagnosed with ischemic stroke. The total sample size was 172 individuals, and the average age was 65 years; they were divided into groups, with the size of each group ranging from seven to 36 individuals. In one study ${ }^{15}$, there was no description of the difference between the intervention and control groups since all of the participants received interventions; the participants were instead divided according to stroke severity, as assessed by the National Institutes of Health Stroke Scale (NIHSS). The other three studies ${ }^{22-24}$ divided the individuals into intervention and control groups. Beer et al. (2018) described the control group as healthy individuals. Two studies $^{22,24}$ included only individuals with one stroke, two evaluated patients within 1 to 10 days of an ischemic stroke $e^{15,23}$, one study evaluated individuals' post-acute stroke ${ }^{24}$, and another study evaluated individuals at 15 days after a stroke ${ }^{22}$. The characteristics of the included studies are shown in Table 1.

All studies evaluated individuals based on the analysis of linear heart rate variables, as shown in Table 2.

Table 2. Variables evaluated in the four included studies.

\begin{tabular}{lc}
\hline HRV & System evaluated \\
\hline SDNN & Sympathetic and parasympathetic activity \\
RMSSD & Parasympathetic activity \\
LF & Sympathetic and parasympathetic activity \\
HF & Parasympathetic activity \\
InHF & Parasympathetic activity \\
LF/HF & Sympathetic and parasympathetic balance \\
\hline
\end{tabular}

HRV: heart rate variability; SDNN: standard deviation of normal R-R intervals; RMSSD: root-mean-square difference of successive normal R-R intervals; LF: low frequency; HF: high frequency; InHF: natural logarithm of HF power; LF/HF: low to high frequency ratio.

\section{Evaluations and interventions}

The interventions reported by the studies were early mobilization ${ }^{15}$, low-intensity activity associated with meditation $^{23}$, cycle ergometer and cognitive activities ${ }^{24}$, and protocol mobilization with a cycle ergometer, which were determined by exercise resistance tests individually (cycle ergometer, 
walking test, and going up and down stairs) ${ }^{22}$. All individuals in the control group performed activities such as conventional physical therapy.

In the study by Nozoe et al. (2018) ${ }^{15}$, the variables LH, InHF, and LF/HF ratio were evaluated by a cardiac monitor, and in the analysis, the complement Lab Chart Pro HRV (ADInstruments Pty Ltd, Castle Hill, Australia) was used. In the intervention protocol, the participants performed an early mobilization in the sitting position; the evaluation comprised 5 minutes in the supine position (rest), followed by five minutes in the sitting position. The patients were reevaluated three months after the stroke.

In the study by Chen et al. (2019) ${ }^{23}$, the variables SDNN, $\mathrm{LF}, \mathrm{HF}$, and LF/HF ratio were evaluated during the execution of Chan-Chuang qigong, known as traditional Chinese medicine therapy, which promotes body-mind interaction and relaxation. The individuals performed the technique for 15 minutes each day for 10 days; the assessment took five minutes and was performed using a portable HRV analyzer (8Z11, Enjoy Research Inc., Taiwan), the Chinese version of the Short Form-12 (SF-12) to assess quality of life, and the Hospital Anxiety and Depression Scale (HADS) to assess negative emotions.

In the study by Beer et al. (2018) ${ }^{24}$, individuals underwent a protocol in which they were first evaluated at rest for 10 minutes, and then they were evaluated during a handgrip activity that lasted two minutes accompanied by controlled breathing (two minutes - six cycles in one minute). Afterwards, they performed cognitive activity (serial 3's subtractions) and finally mobilization with a cycle ergometer in combination with a cognitive exercise. Cognitive capacity was assessed using the Montreal Cognitive Assessment Scale (MoCA), and the Barthel index was used to assess functional capacity. The variables SDNN and RMSSD were measured by the Polar Advanced Heart Rate Monitor (RS800CX).

All included studies performed evaluations of linear heart rate variables; however, studies did not present heterogeneity among the groups, interventions, or evaluations. Only one study, of the four included, did not show significant results in relation to the variables evaluated. All studies excluded individuals who had heart disease.

\section{Evaluation of the effectiveness and safety of the included studies}

The evaluation of the effectiveness and safety of the included studies are displayed in the Table 3.

In the study by Nozoe et al. (2018) ${ }^{15}$, there were no significant differences in the InHF between the intervention and rest values for the non-neurological deterioration (ND) group $=4.0(3.2,5.2)$ or ND group $=4.7(4,5) ; P=0.74$; the $\mathrm{LF} / \mathrm{HF}$ ratios were as follows: non-ND group $=1.9(0.5,3.2)$, ND group $=1.0(0.8,3.3) ; P=0.91$. During mobilization, there were no significant differences in InHF between the non-ND group = $4.9(3.3,5.9)$ and ND group $=4.6(3.9,4.9) ; P=0.74$. However, the LF/HF ratio was significantly higher in the ND group = 1.7 (SD 0.9, 2.6) than in the non-ND group $=0.6(0.4,1.5) ; P$ $=0.03$. The authors did not report any adverse effects after intervention.

In the study by Chen et al. (2019) ${ }^{23}$, the LF/HF ratio was higher in the intervention group after early mobilization regarding the physical component of the quality of life (QOL) scale (SF-12) than in the control group $(P=0.02)$. The authors did not report the effect sizes or confidence intervals of the data, and any adverse effects were observed after intervention.

Table 3. Interventions, results, and GRADE of the included studies.

\begin{tabular}{|c|c|c|c|}
\hline Author (year) & Interventions & Results & GRADE \\
\hline Nozoe et al., $2018^{15}$ & $\begin{array}{l}\text { Early mobilization in the ND group }(n=7) \\
\text { and early mobilization in the non-ND group } \\
\qquad(n=14)\end{array}$ & $\begin{array}{l}\text { LF/HF is higher in patients with ND } \\
\text { during early mobilization; suggests } \\
\text { increase in sympathetic activity }\end{array}$ & $\stackrel{\oplus}{\text { Very Low }}$ \\
\hline Chen et al., $2019^{23}$ & $\begin{array}{l}\text { Mind-body interactive exercise - } \\
\text { Chan-Chuang qigong practice (15 minutes/ } \\
\text { day) }(n=36) \text { and control group }(n=36)\end{array}$ & $\begin{array}{l}\text { LF/HF ratio significantly influenced the } \\
\text { physical component of quality of life; } \\
\text { suggests cardiac autonomic balance } \\
\text { after intervention }\end{array}$ & $\begin{array}{l}\oplus \oplus \\
\text { LoW }\end{array}$ \\
\hline Beer et al., $2018^{24}$ & $\begin{array}{l}\text { Static and dynamic exercises; dual task; } \\
\text { breathing exercise for } 2 \text { minutes. }\end{array}$ & $\begin{array}{l}\text { Resting SDNN was significantly lower } \\
\text { among post-stroke patients compared } \\
\text { with healthy individuals; less adaptive } \\
\text { cardiac autonomic control during } \\
\text { different activities }\end{array}$ & $\begin{array}{c}\oplus \\
\text { Very Low }\end{array}$ \\
\hline $\begin{array}{l}\text { Katz-Leurer \& } \\
\text { Shochina, 200722 }\end{array}$ & Conventional physical therapy & No alterations in HRV & $\begin{array}{l}\oplus \oplus \\
\text { Low }\end{array}$ \\
\hline
\end{tabular}

ND: neurological deterioration classified by the NIHSS; LF: Low frequency; HF: high frequency; LF/HF: low to high frequency ratio; SDNN: standard deviation of normal R-R intervals; HRV: heart rate variability. GRADE classification: - High-quality evidence: Findings are consistent among at least $75 \%$ of the RCTs with a low risk of bias; data are consistent, direct, and precise, and no publication biases are known or suspected. Additional research is unlikely to change the estimate or our confidence in the results; - Moderate-quality evidence: One of the domains is not met. Additional research is likely to have an important impact on our confidence in the estimate of effect and may change the estimate; $\bullet$ Low-quality evidence: Two of the domains are not met. Additional research is very likely to have an important impact on our confidence in the estimate of effect and is likely to change the estimate; • Very low-quality evidence: Three of the domains are not met. We are very uncertain about the results. 
The study by Beer et al. (2018) ${ }^{24}$ showed less adaptive cardiac autonomic control during different activities. The values described for the groups were as follows: post stroke RR $=728.7 \pm 110.1 \mathrm{~ms}$; healthy individuals $\mathrm{RR}=847.6 \pm 120.6 \mathrm{~ms}$, with $P=0.002$; post-stroke SDNN $=32.5 \pm 26.9 \mathrm{~ms}$, healthy individuals SDNN $=48.7 \pm 17.9 \mathrm{~ms}$, with $P=0.01$. The authors did not report any adverse effects after intervention.

In the study by Katz-Leurer and Shochina (2007) ${ }^{22}$, no significant interaction effects on HRV were observed between exercises during physical therapy. The values indicated for the variables were as follows: treatment group LF $=1248 \pm 1684$
$\mathrm{Hz}$, control group LF $=1238 \pm 1728 \mathrm{~Hz}$, with $P=0.93$; treatment group $\mathrm{HF}=378 \pm 638 \mathrm{~Hz}$, control group $\mathrm{HF}=667 \pm 150$ $\mathrm{Hz}$, with a $P=0.33$. The authors did not report any adverse effects after intervention.

\section{Risk of bias interpretation}

All included articles were analyzed for risk of bias, as shown in Table 4.

Figure 2 shows a graphical analysis of the risk of bias.

Table 4. Risk of bias classification

\begin{tabular}{|c|c|c|c|}
\hline Risk of bias & High Risk & Low Risk & Uncertain Risk \\
\hline $\begin{array}{l}\text { Random sequence } \\
\text { generation }\end{array}$ & Beer et al., 2018²4; Nozoe et al., $2018^{15}$ & $\begin{array}{l}\text { Chen et al., } 2019^{23} \text {; } \\
\text { Katz-Leurer; Shochina, } 2007^{22}\end{array}$ & None \\
\hline $\begin{array}{l}\text { Allocation } \\
\text { concealment }\end{array}$ & Beer et al., 2018²4; Nozoe et al., $2018^{15}$ & Chen et al., $2019^{23}$ & Katz-Leurer; Shochina, $2007^{22}$ \\
\hline $\begin{array}{l}\text { Blinding of the } \\
\text { participants }\end{array}$ & Chen et al., $2019^{23}$ & None & $\begin{array}{l}\text { Katz-Leurer; Shochina, } 2007^{22} \\
\text { Beer et al., 2018²; Nozoe et al., } 2018^{15}\end{array}$ \\
\hline $\begin{array}{l}\text { Blinding of the } \\
\text { outcome } \\
\text { assessment }\end{array}$ & Chen et al., $2019^{23}$ & None & $\begin{array}{l}\text { Katz-Leurer; Shochina, } 2007^{22} \\
\text { Beer et al., 2018 } \\
\text { 24; Nozoe et al., } 2018^{15}\end{array}$ \\
\hline $\begin{array}{l}\text { Incomplete outcome } \\
\text { data }\end{array}$ & Beer et al., 2018²4; Nozoe et al., $2018^{15}$ & Chen et al., $2019^{23}$ & Katz-Leurer; Shochina, $2007^{22}$ \\
\hline $\begin{array}{l}\text { Selective outcome } \\
\text { reporting }\end{array}$ & None & Chen et al., $2019^{23}$ & $\begin{array}{l}\text { Katz-Leurer; Shochina, } 2007^{22} \\
\text { Beer et al., 2018²; Nozoe et al., } 2018^{15}\end{array}$ \\
\hline
\end{tabular}

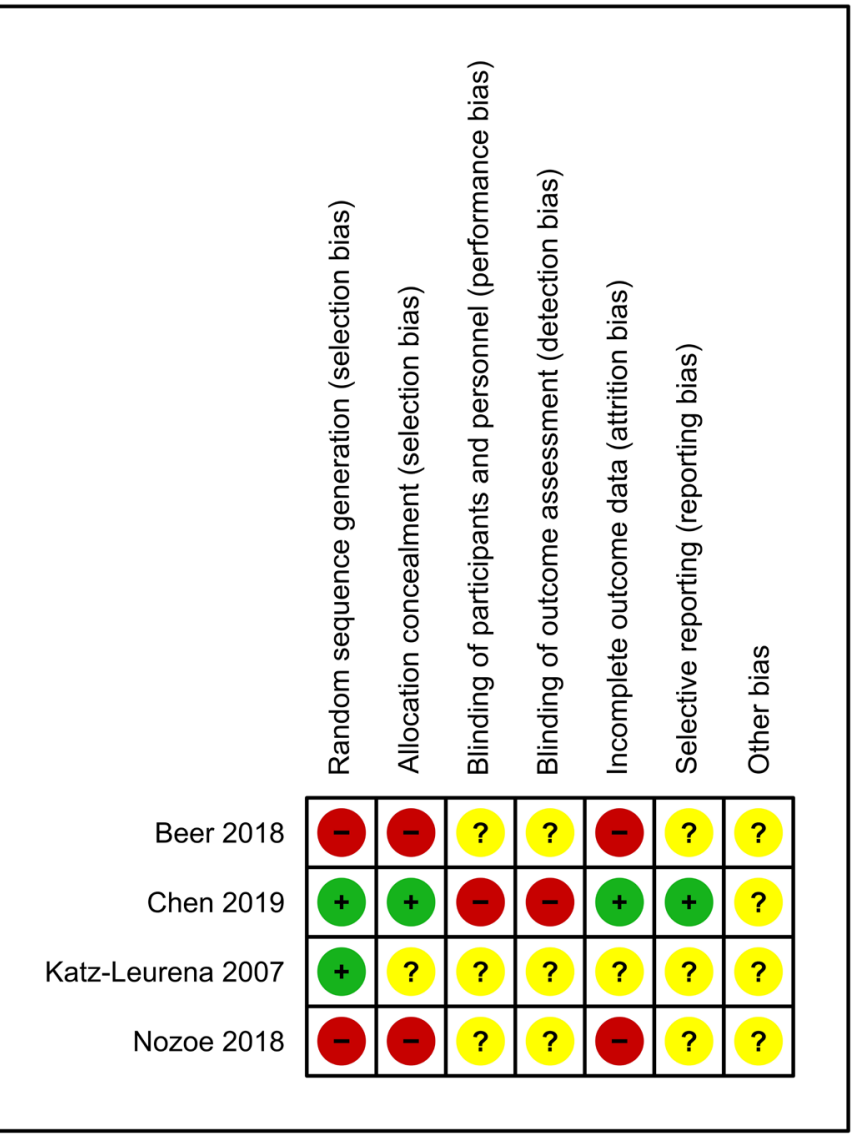

Red: high; green: low; yellow: uncertain.

Figure 2. Graphical analysis of the risk of bias in the included studies. 


\section{DISCUSSION}

This systematic literature review study comprised four articles from clinical trials that aimed to assess HRV using different methodologies, describing sympathovagal activity after specific rehabilitation protocols in patients after ischemic stroke.

Of the four studies included, two ${ }^{22,24}$ used the cycle ergometer for the main rehabilitation program. Only the study by Beer et al. (2018) ${ }^{24}$ showed a significant reduction in the RR and SDNN variables among post-stroke individuals compared to healthy individuals at rest, which indicates a state of sympathetic hyperactivity in the subacute phase after the stroke. In this study, patients did not show a normal increase in sympathetic activity in response to the test conditions, mainly due to a hypersympathetic state at rest. During the subacute phase, according to this study and other studies ${ }^{25,26}$, there is apparently a significant physiological change in the ability of the autonomic nervous system to respond adequately to the demands imposed by rehabilitation practices, so only large demands yield expected sympathetic responses ${ }^{27}$. The results indicate a need for rehabilitation focused on improving autonomic cardiac control.

In the study by Nozoe et al. $(2018)^{15}$, patients were classified as having or not neurological deterioration (ND) using the NIHSS score (severity scale used in the acute phase of stroke). These individuals were evaluated during hospitalization and underwent an intervention involving early mobilization with posture changes. The LF/HF ratio showed a significant increase in the ND group (a higher NIHSS score) from before to after the intervention. Since the LF/HF ratio seems to reflect sympathetic performance, according to the authors, it is likely that an increase in sympathetic activity during mobilization is associated with neurological deterioration in acute stroke patients. Xiong et al. $(2018)^{28}$ reported that autonomic dysfunction is one of the predictors of worse functional outcomes in patients in the acute phase of stroke, which can confirm the possible occurrence of increased sympathetic performance in patients with a worse NIHSS classification.

Chen et al. (2019) ${ }^{23}$ introduced a mind-body interactive exercise (Chan-Chuang qigong practice) as an intervention for hospitalized patients after stroke to increase cardiac parasympathetic tone mainly because the technique has relaxing effects. They concluded that the LF/HF ratio regarding the physical component of the quality of life (QOL) scale (SF12) was higher in the intervention group after mobilization than in the control group. Therefore, during the hospital stay, the sympathovagal balance influenced the physical aspect of the QOL of individuals with subacute stroke. Thus, improved HRV in stroke patients after a specific rehabilitation protocol can lead to the recovery of physical functions and improve their quality of life.

In the study by Katz-Leurer and Shochina (2007) $)^{22}$, an individualized training protocol was used, and they did not find significant differences in HRV. Despite this result, a significant improvement was found in the functional parameters of post-stroke individuals, such as climbing stairs, and physical training allowed patients to significantly increase their workload. As described by other authors, autonomic impairment after stroke leads to low aerobic capacity ${ }^{27}$. Thus, the importance of early mobilization, rehabilitation, and physical-functional training in post-stroke patients is reiterated.

The authors reported sympathetic-vagal alterations in poststroke patients when subjected to physical activities. Thus, from this systematic review, it can be stated that significant autonomic modulation occurs in these individuals. Despite the methodological divergence found in the articles, only one article reported no changes in HRV between the groups evaluated ${ }^{22}$, which established an assessment in the frequency domain. In the study by Beer et al. $(2018)^{24}$, variables in the time domain were included, whereas assessments in both domains (time and frequency) were included in other studies, which demonstrated significant changes in the HRV linear variables after stroke rehabilitation.

Studies on HRV demonstrate the need for flexibility in autonomic activity for individuals to maintain a good quality of life, as impaired adaptation can cause autonomic dysfunctions, cardiovascular deterioration, and increased morbidity and mortality rates in patients after stroke ${ }^{28}$. The four articles selected for the review show the need for specific therapies, early mobilizations, and physical activity protocols in the modulation of HRV. This conclusion points to the importance of maintaining muscle function, strength, and activity for cardiovascular benefits, which has been widely studied for methods including cardiac rehabilitation ${ }^{28-30}$.

This study has limitations, such as heterogeneity in the selected individuals and the analyzed outcomes; because only a few studies were selected, it was impossible to perform a meta-analysis. However, this is the first systematic review addressing this topic, with the possibility of elucidating the main autonomic repercussions observed in post-stroke patients undergoing rehabilitation procedures.

In conclusion, thequality of the evidence from the selected clinical trials was either low or very low; therefore, there are no definitive conclusions about the main autonomic repercussions observed in post-stroke patients undergoing rehabilitation, although all interventions are safe for these patients. The applicability of these results can be compromised since most of the results described in this review were obtained from clinical trials with methodological differences. This review highlights the need to conduct well-designed tests in this field. Future trials should be properly designed and should include standardized measures. It is suggested that RCTs address a heterogeneous population and include measures in the time and frequency domains, in addition to a nonlinear analysis of HR, to establish parameters of sympathetic-vagal behavior during rehabilitation protocols after stroke. 
1. Mehta RH, Eagle K. Secondary prevention in acute myocardial infarction. BMJ. 1998 Mar 14;316(7134):838-42. https://doi. org/10.1136/bmj.316.7134.838

2. Powers WJ, Rabinstein AA, Ackerson T, Adeoye OM, Bambakids NC, Becker K, et al. 2018 Guidelines for the early management of patients with acute ischemic stroke: a guideline for healthcare professionals from the American Heart Association/American Stroke Association. Stroke. 2018 Mar;49(3):e46-110. https://doi.org/10.1161/ STR.0000000000000158

3. Johnston SC, Mendis S, Mathers CD. Global variation in stroke burden and mortality: estimates from monitoring, surveillance, and modelling. Lancet Neurol. 2009 Apr;8(4):345-54. https://doi. org/10.1016/\$1474-4422(09)70023-7

4. Thayer JF, Yamamoto SS, Brosschot JF. The relationship of autonomic imbalance, heart rate variability and cardiovascular disease risk factors. Int J Cardiol. 2010 May 28;141(2):122-31. https://doi. org/10.1016/j.ijcard.2009.09.543

5. Keenan NL, Shaw KM, Centers for Diseade Control and Prevention (CDC). Coronary heart disease and stroke deaths - United States, 2006. MMWR Suppl. 2011 Jan 14;60(1):62-6.

6. Winstein CJ, Stein J, Arena R, Bates B, Cherney LR, Cramer SC, et al. Guidelines for adult stroke rehabilitation and recovery. Stroke. 2016 May 4;47(6):e98-169. https://doi.org/10.1161/ STR.0000000000000098

7. Vanderlei LCM, Pastre CM, Hoshi RA, Carvalho TD, Godoy MF. Noções básicas de variabilidade da frequência cardíaca e sua aplicabilidade clínica. Rev Bras Cir Cardiovasc. 2009 Set 14;24(2):205-17. https:// doi.org/10.1590/S0102-76382009000200018

8. Nussinovitch U, Katz U, Nussinovitch M, Nussinovitch N. Deep breath test for evaluation of autonomic nervous system dysfunction in familial dysautonomia. Isr Med Assoc J. 2009 Oct;11(10):615-8.

9. Archiza B, Simões RP, Mendes RG, Fregonezi GAF, Catai AM, BorghiSilva A. Acute effects of different inspiratory resistive loading on heart rate variability in healthy elderly patients. Braz J Phys Ther. 2013 Aug 23;17(4):401-8. https://doi.org/10.1590/S141335552013005000100

10. Hilz MJ, Moeller S, Akhundova A, Marthol H, Pauli E, De Fina P, et al. NIHSS values predict impairment of cardiovascular autonomic control. Stroke. 2011 Jun;42(6):1528-33. https://doi.org/10.1161/ STROKEAHA.110.607721

11. Thayer JF, Lane RD. The role of vagal function in the risk for cardiovascular disease and mortality. Biol Psychol. 2007 Feb;74(2):224-42. https://doi.org/10.1016/j.biopsycho.2005.11.013

12. Xu Y-H, Wang X-D, Yang J-J, Zhou L, Pan Y-C. Changes of deceleration and acceleration capacity of heart rate in patients with acute hemispheric ischemic stroke. Clin Interv Aging. 2016 Mar 11;11:293-8. https://doi.org/10.2147/CIA.S99542

13. Shields RW Jr. Heart rate variability with deep breathing as a clinical test of cardiovagal function. Cleve Clin J Med. 2009 Apr;76 (4 Suppl 2):S37-40. https://doi.org/10.3949/ccjm.76.s2.08

14. Harunobu U, Yuusuke N. Relationship between physical activity and the very low-frequency component of heart rate variability after stroke. J Stroke Cerebrovasc Dis. 2015 Apr;24(4):840-3. https://doi. org/10.1016/j.jstrokecerebrovasdis.2014.11.026

15. Nozoe M, Yamamoto M, Kobayashi M, Kanai M, Kubo H, Shimada S, et al. Heart rate variability during early mobilization in patients with acute ischemic stroke. Eur Neurol. 2018;80(1-2):50-4. https://doi. org/10.1159/000492794

16. Prosser J, MacGregor L, Lees KR, Diener H-C, Hacke W, Davis S,
VISTA Ivestigator. Predictors of early cardiac morbidity and mortality after ischemic stroke. Stroke. 2007 Aug;38(8):2295-302. https://doi. org/10.1161/STROKEAHA.106.471813

17. Bassi A, Colivicchi F, Santini M, Caltagirone C. Cardiac autonomic dysfunction and functional outcome after ischaemic stroke. Eur J Neurol. 2007 Aug;14(8):917-22. https://doi.org/10.1111/j.14681331.2007.01875.x

18. Higgins JPT, Green S, editors. Cochrane handbook for systematic reviews of interventions Version 5.1.0 [Internet]. The Cochrane Collaboration; 2011. Available from: https://handbook-5-1.cochrane. org/

19. Shamseer L, Moher D, Clarke M, Ghersi D, Liberati A, Petticrew M, et al. Preferred reporting items for systematic review and meta-analysis protocols (PRISMA-P): elaboration and explanation. BMJ. $2016 \mathrm{Jul}$ 21;354:i4086. https://doi.org/10.1136/bmj.i4086

20. Higgins JP, Altman DG, Gøtzsche PC, Jüni P, Moher D, Oxman AD, Savovic J, et al. The cochrane collaboration's tool for assessing risk of bias in randomised trials. BMJ. 2011 Oct 18;343:d5928. https://doi. org/10.1136/bmj.d5928

21. Guyatt GH, Oxman AD, Vist GE, Kunz R, Falck-Ytter Y, Alonso-Coello P, et al. GRADE: An emerging consensus on rating quality of evidence and strength of recommendations. BMJ. 2008 Apr 26;336(7650):9246. https://doi.org/10.1136/bmj.39489.470347.AD

22. Katz-Leurer M, Shochina M. The influence of autonomic impairment on aerobic exercise outcome in stroke patients. NeuroRehabilitation. 2007 Oct;22(4):267-72. https://doi.org/10.3233/NRE-2007-22403

23. Chen $\mathrm{CH}$, Hung KS, Chung YC, Yeh ML. Mind-body interactive qigong improves physical and mental aspects of quality of life in patients with stroke: a randomized control study. Eur J Cardiovasc Nurs. 2019 Dec;18(8):658-66. https://doi.org/10.1177/1474515119860232

24. Beer NR, Soroker N, Bornstein N, Katz-Leurer M. The cardiac autonomic nervous system response to different daily demands among patients at the sub-acute phase post ischemic stroke and healthy controls. NeuroRehabilitation. 2018;42(4):391-6. https://doi. org/10.3233/NRE-172295

25. Katz-Leurer M, Zohar M, Boum A, Keren O. Monitoring changes in heart rate, as an indicator of the cardiovascular automatic nervous function, among patients at the sub-acute phase post-brain damage during a physiotherapy session: a preliminary investigation. Brain Inj. 2014;28(1):127-31. https://doi.org/10.3109/02699052.2013.848381

26. Raimundo DR, De Abreu LC, Adami F, Vanderlei FM, de Carvalho TD, Moreno IL, et al. Heart rate variability in stroke patients submitted to an acute bout of aerobic exercise. Transl Stroke Res. 2013 Oct;4(5):488-99. https://doi.org/10.1007/s12975-013-0263-4

27. Beer NR, Bornstein NM, Soroker N, Katz-Leurer M. Autonomic cardiac response to static and dynamic muscle contractions in post-stroke and healthy subjects. Eur Neurol. 2016;75(5-6):207-12. https://doi. org/10.1159/000445769

28. Xiong L, Tian G, Leung H, Soo YOY, Chen X, Ip VHL, et al. Autonomic dysfunction predicts clinical outcomes after acute ischemic stroke: a prospective observational study. Stroke. 2018 Jan;49(1):215-8. https://doi.org/10.1161/STROKEAHA.117.019312

29. Gregoire J, Tuck S, Hughson RL, Yamamoto Y. Heart rate variability at rest and exercise: influence of age, gender, and physical training. Can J Appl Physiol. 1996 Dec;21(6):455-70. https://doi.org/10.1139/ h96-040

30. De Raedt S, De Vos A, De Keyser J. Autonomic dysfunction in acute ischemic stroke: an underexplored therapeutic area? J Neurol Sci. 2015 Jan 15;348(1-2):24-34. https://doi.org/10.1016/j. jns.2014.12.007 